\title{
Effects of water domains on seismic wavefields: A simulation case study at Taal volcano, Philippines
}

\author{
Yuta Maeda and Hiroyuki Kumagai \\ National Research Institute for Earth Science and Disaster Prevention, 3-1 Tennodai, Tsukuba, Ibaraki 305-0006, Japan
}

(Received September 9, 2011; Revised July 10, 2012; Accepted July 10, 2012; Online published March 6, 2013)

\begin{abstract}
We examined the effects of water domains on seismic wave elds at the Taal volcano, Philippines, where there is a summit crater lake on an active volcanic island surrounded by a caldera lake. We conducted forward waveform simulation and synthetic waveform inversion using the 3D nite-difference method for various models with and without the water lakes. Our study demonstrated the existence of both near- and far- eld effects of the water domains. The near- eld effect is related to source excitation affected by a water domain, and the far- eld effect is caused by dispersion of Rayleigh waves propagating through a water domain. Our study also showed that the replacement of water by a vacuum rather than by a solid medium provides a better approximation of a water domain. A water domain has considerable effect on seismic waveforms and must be properly treated when the waveform period is shorter than $2 \mathrm{~s}$, even if recording stations lie in the near eld. If the waveform period is longer than $5 \mathrm{~s}$, a water domain has a minor effect on seismic waveforms and may be replaced by a vacuum.

Key words: Volcano seismology, moment tensor inversion, nite-difference method, Taal volcano.
\end{abstract}

\section{Introduction}

Long-period (LP: 0.2-2 s) and very-long-period (VLP: 2-100 s) seismic signals are commonly observed at volcanoes worldwide. They are thought to be generated by volumetric changes and movements of volcanic uids, and are thus important for understanding pre-eruptive uid processes in volcanoes. To quantify LP and VLP sources, waveform inversion techniques developed by Ohminato et al. (1998), Nakano and Kumagai (2005), and Auger et al. (2006) have been commonly used. In waveform inversion analyses, accurate estimations of Green's functions are critically important.

Waveform inversion studies have been conducted at volcanoes with crater lakes and volcanic islands surrounded by sea water, such as Kilauea (Ohminato et al., 1998), Aso (Legrand et al., 2000), Miyake (Kumagai et al., 2001; Kobayashi et al., 2009), Kusatsu-Shirane (Nakano et al., 2003), Usu (Yamamoto et al., 2002), Stromboli (Chouet et al., 2003), Hachijo (Kumagai et al., 2003), and SatsumaIwojima (Ohminato, 2006). In these studies, water domains were usually ignored and represented either by solid or vacuum domains in calculations of Green's functions, but without stated justi cation except for the study of Chouet $e t$ al. (2003), who concluded that the effect of sea water was negligible within a VLP band at Stromboli.

The in uence of water domains on synthetic seismograms has been more extensively studied in strong ground motion seismology. Using a 2D boundary element method, Hatayama (2004) synthesized Rayleigh waves at an epicen-

Copyright (c) The Society of Geomagnetism and Earth, Planetary and Space Sciences (SGEPSS); The Seismological Society of Japan; The Volcanological Society of Japan; The Geodetic Society of Japan; The Japanese Society for Planetary Sciences; TERRAPUB.

doi:10.5047/eps.2012.07.004 tral distance of $60 \mathrm{~km}$ from a seismic source at a depth of $5 \mathrm{~km}$ with, and without, including a sea layer. He concluded that the sea has an important effect on the Rayleigh wave if the source duration is shorter than $3 \mathrm{~s}$ and the water depth is greater than $800 \mathrm{~m}$. He also concluded that the replacement of sea water by a vacuum provides a better approximation for the sea layer than does the substitution of a solid medium for sea water. Petukhin et al. (2010) used a 3D nite-difference method to calculate synthetic seismograms of large interplate earthquakes by using a 3D velocity and density structure with, and without, an oceanic layer. They concluded that the oceanic layer has a large in uence on the Rayleigh waves for a shallow ( $\sim 5-\mathrm{km}$ depth) earthquake, whereas it has negligible in uence on synthetic seismograms for a deep (>10-km depth) source.

Although these strong-motion studies considered earthquake sources at depths greater than $5 \mathrm{~km}$, LP and VLP events at volcanoes often occur at depths of $1 \mathrm{~km}$ or shallower. Seismic stations at volcanoes are located at source distances from a few hundred meters to several kilometers. Volcanoes exhibit steep and complex topographies. The effects of water domains on seismograms at volcanoes in such complex situations may depend on station con gurations and the periods, or wavelengths, of LP and VLP signals, and should be evaluated by numerical simulation approaches.

Taal volcano, Philippines, is $60 \mathrm{~km}$ south of metropolitan Manila (Fig. 1(a)). Since 1572, 33 known phreatic and phreatomagmatic explosions at Taal have killed a total of more than 1500 people (Zlotnicki et al., 2009). Most of the Taal eruptions have taken place after quiescent periods of less than 30 years, but more than 30 years have passed since the last eruption in 1977. This implies a high risk of nearfuture eruptions at Taal. To improve the monitoring and understanding of the volcano, a joint Japan-Philippine team 

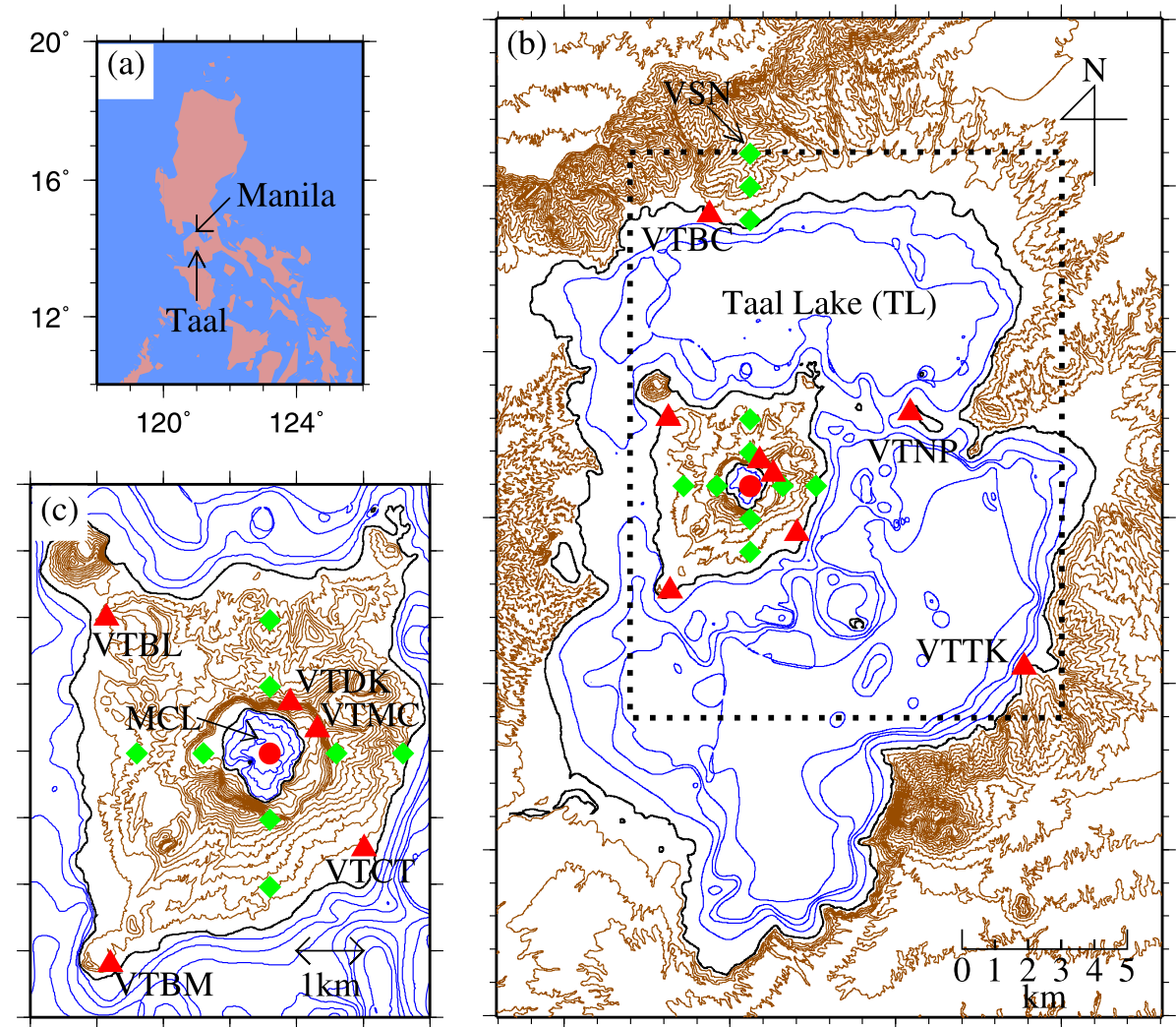

Fig. 1. (a) Location of Taal volcano, Philippines. (b) Map showing source and station locations. Red circle represents the epicenter. Red triangles indicate real station locations, and green diamonds indicate the locations of virtual stations (at 1-km intervals from the epicenter) used in the forward waveform simulation. The dotted rectangle shows the extent of the horizontal computational domain. Contours indicate the topography of the Taal volcano and bathymetries of Taal Lake (TL) and Main Crater Lake (MCL). The contour interval is $40 \mathrm{~m}$. (c) Enlargement of map (b) around the Volcano Island. The contour interval is $20 \mathrm{~m}$.

installed five broadband seismometers at Taal in November 2010, as well as infrasonic and geomagnetic sensors and GPS receivers.

Taal holds a caldera filled with water (Taal Lake: TL), which has EW and NS dimensions of 15 and $25 \mathrm{~km}$, respectively, and a maximum depth of $200 \mathrm{~m}$ (Fig. 1(b)). The currently-active crater is the Volcano Island (elevation 311 m) surrounded by TL. The active crater forms the Main Crater Lake (MCL) with a diameter of $1.2 \mathrm{~km}$ and a maximum water depth of $80 \mathrm{~m}$ (Fig. 1(c)). Taal thus provides a good test field to evaluate the effects of water domains on seismic waveforms. Such evaluations are important for the correct calculations of Green's functions, which are used in broadband seismic monitoring at Taal based on the waveform inversion approach.

In this study, we evaluated the effects of the two water domains on seismic wavefields at Taal volcano through forward waveform simulation and synthetic waveform inversion. We calculated synthetic seismograms using the finitedifference method, assuming the following three models: the water domains treated as water (water model), the water replaced by a vacuum (vacuum model), and a solid medium (solid model). In Section 2, we describe the finitedifference method used to calculate synthetic seismograms. In Section 3, we compare forward synthetic seismograms calculated for the three models at various epicentral distances to evaluate the effects of the water domains on the seismic wavefields. Then, in Section 4 , using synthetic ob- served seismograms and Green's functions at real seismic station locations, we examine the effects of the water domains on waveform inversion. We discuss our results in Section 5, and present our conclusions in Section 6.

\section{Calculation of Synthetic Seismograms}

We used a second-order finite-difference method (FDM) to calculate synthetic seismograms. In the FDM code, we used algorithms proposed by Maeda et al. (2011), and Okamoto and Takenaka (2005). The algorithm of Maeda et al. (2011) uses the staggered grid cell proposed by Ohminato and Chouet (1997) to calculate the synthetic seismograms for models with arbitrary 3D topography and structure. An efficient absorbing boundary, known as a perfectly matched layer (PML; Chew and Liu, 1996; Festa and Nielsen, 2003), is used to efficiently calculate long synthetic seismograms. The algorithm of Okamoto and Takenaka (2005) allows the inclusion of a water domain, which is realized by setting Lame's coefficient $\mu$ to zero for the cells representing the water domain. The solid-water boundary is placed at the interfaces of the grid cells, where $\mu$ is set to zero, so that the tangential stress is zero and the normal velocity is continuous at the boundary. An average of the solid and water densities is used as the density at the solidwater boundary. Okamoto and Takenaka (2005) theoretically showed that the normal stress component is continuous through the boundary when the averaged density is used in the second-order finite-difference equations. Okamoto 


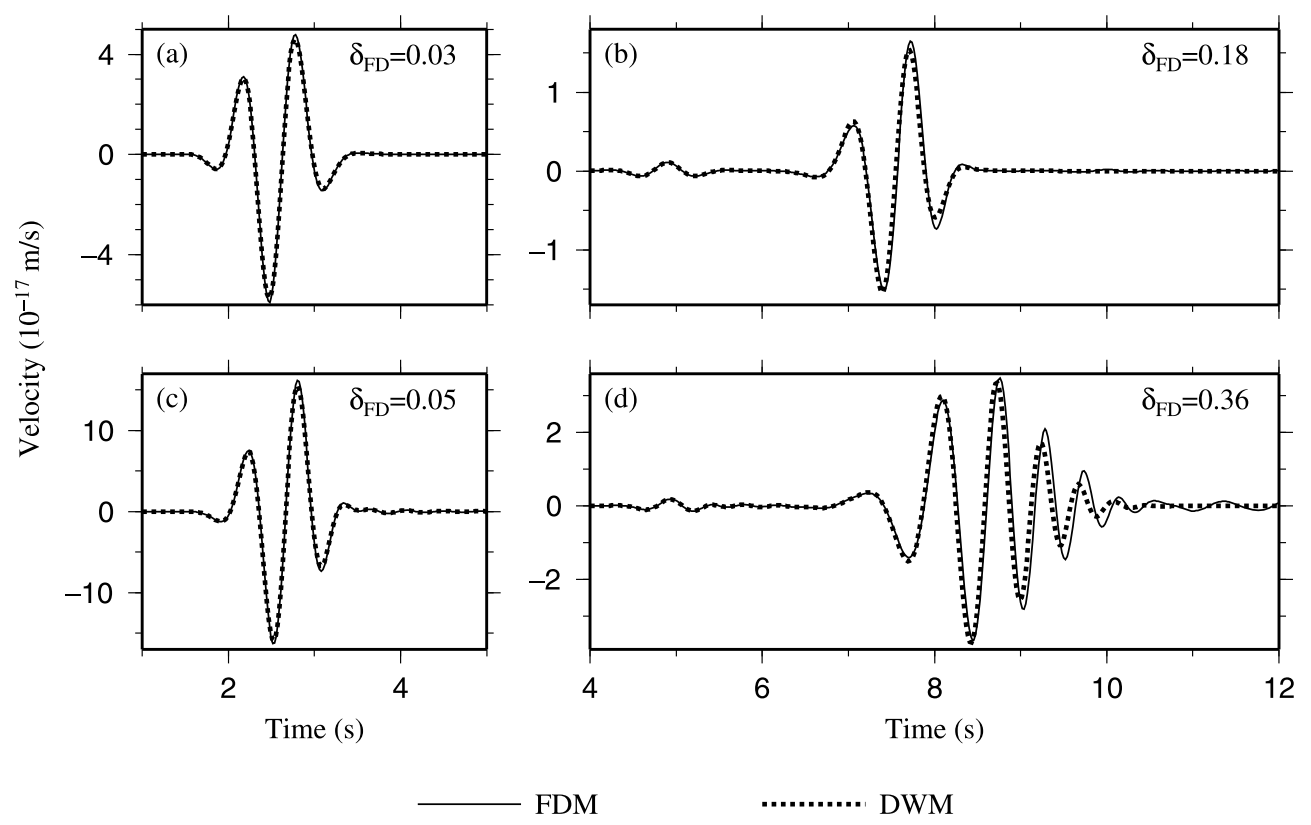

Fig. 2. Vertical velocity waveforms on water surfaces calculated with a structure composed of a flat water layer overlying a homogeneous half space. Solid and dotted lines indicate the waveforms calculated using FDM and DWM codes, respectively. Waveforms calculated with a water depth of 40 $\mathrm{m}$ (1 grid cell) at epicentral distances of $1 \mathrm{~km}$ (a) and $10 \mathrm{~km}$ (b) and a water depth of $160 \mathrm{~m}$ (4 grid cells) at epicentral distances of $1 \mathrm{~km}$ (c) and 10 $\mathrm{km}(\mathrm{d})$ are shown.

and Takenaka (2005), and Nakamura et al. (2011), used numerical tests to justify their use of these boundary conditions. We used Cartesian coordinates $x, y$, and $z$ corresponding to E, N, and upward, respectively, throughout this study.

To evaluate the accuracy of the FDM code, we performed a simulation using a flat water layer overlying a homogeneous solid half space. For the water layer, we assumed a $P$-wave velocity $V_{p}=1450 \mathrm{~m} / \mathrm{s}$, an $S$-wave velocity $V_{s}=0 \mathrm{~m} / \mathrm{s}$, and a density $\rho=1020 \mathrm{~kg} / \mathrm{m}^{3}$. For the solid half space, we assumed $V_{p}=3500 \mathrm{~m} / \mathrm{s}, V_{s}=V_{p} / \sqrt{3}$, and $\rho=2400 \mathrm{~kg} / \mathrm{m}^{3}$. We used the following Ricker wavelet as the input source time function,

$$
\begin{gathered}
f(t)=(\sqrt{\pi} / 2)\left[b(t)^{2}-1 / 2\right] \exp \left[-b(t)^{2}\right], \\
b(t)=\pi\left(t-T_{s}\right) / T_{p},
\end{gathered}
$$

where $t$ is time, and $T_{p}$ and $T_{s}$ are constants related to the duration and peak time of the wavelet, respectively. We used an isotropic source with $T_{p}=1 \mathrm{~s}$ and $T_{s}=2 \mathrm{~s}$ at a depth of $220 \mathrm{~m}$ below the water surface. Figure 2 compares the vertical velocities on the water surface calculated by the FDM code (solid lines), using a uniform $40 \mathrm{~m}$ grid and a time step of $0.004 \mathrm{~s}$, with those calculated by the discrete wavenumber method (DWM) code of Takeo (1985) (dotted lines). In the DWM calculation, we used a small value of an $S$-wave velocity for the water layer $\left(V_{s}=0.01 \mathrm{~m} / \mathrm{s}\right)$ because the DWM code could not treat $V_{s}=0 \mathrm{~m} / \mathrm{s}$. We used waterlayer thicknesses of $40 \mathrm{~m}$ (Figs. 2(a) and 2(b)) and $160 \mathrm{~m}$ (Figs. 2(c) and 2(d)), which are the representative depths of MCL and TL, respectively. Note that the 40-m-thick water-layer is expressed by only one grid cell in the FDM calculation. In Fig. 2, we also indicate misfits among the waveforms, defined as

$$
\delta_{\mathrm{FD}}=\sqrt{\frac{\sum_{k=0}^{N-1}\left[V_{z}^{\mathrm{FDM}}(k \Delta t)-V_{z}^{\mathrm{DWM}}(k \Delta t)\right]^{2}}{\sum_{k=0}^{N-1}\left[V_{z}^{\mathrm{DWM}}(k \Delta t)\right]^{2}}}
$$

where $V_{z}^{\text {FDM }}$ and $V_{z}^{\text {DWM }}$ denote vertical velocity waveforms calculated by the FDM and DWM codes, respectively, $N$ denotes the number of data samples in each trace, and $\Delta t$ denotes a time step $(0.004 \mathrm{~s})$. We obtained the misfit value of 0.03 for an epicentral distance of $1 \mathrm{~km}$ and a water-layer thickness of $40 \mathrm{~m}$ (Fig. 2(a)), and 0.36 for an epicentral distance of $10 \mathrm{~km}$ and a water-layer thickness of $160 \mathrm{~m}$ (Fig. 2(d)). These misfit values will be used to evaluate our synthetic results later.

\section{Forward Synthetic Tests}

In our forward waveform simulation tests, we assumed an isotropic Ricker wavelet (Eqs. (1) and (2)) source beneath the MCL (Fig. 1(c)). We calculated synthetic seismograms at real and virtual station locations (Figs. 1(b) and 1(c)). We used a 10-m mesh digital elevation model (DEM) containing bathymetries of TL and MCL. Delmelle et al. (1998) estimated the water surfaces of TL and MCL to be 2 and $4 \mathrm{~m}$ above sea level, respectively; we therefore set them at sea level. We used the computational domain down to a depth of $4 \mathrm{~km}$ below sea level, surrounded by 800 -m-thick PML domains. We used the same grid size, time step, $P$ and $S$-wave velocities, and densities of the water and solid medium as those in the previous section.

Figure 3 displays velocity snapshots just beneath the land and water surfaces calculated for the water model. To obtain the snapshots covering TL, we used the entire domain of Fig. 1(b) as the horizontal range of the computational domain. The TL shorelines are clearly visible in the snap- 


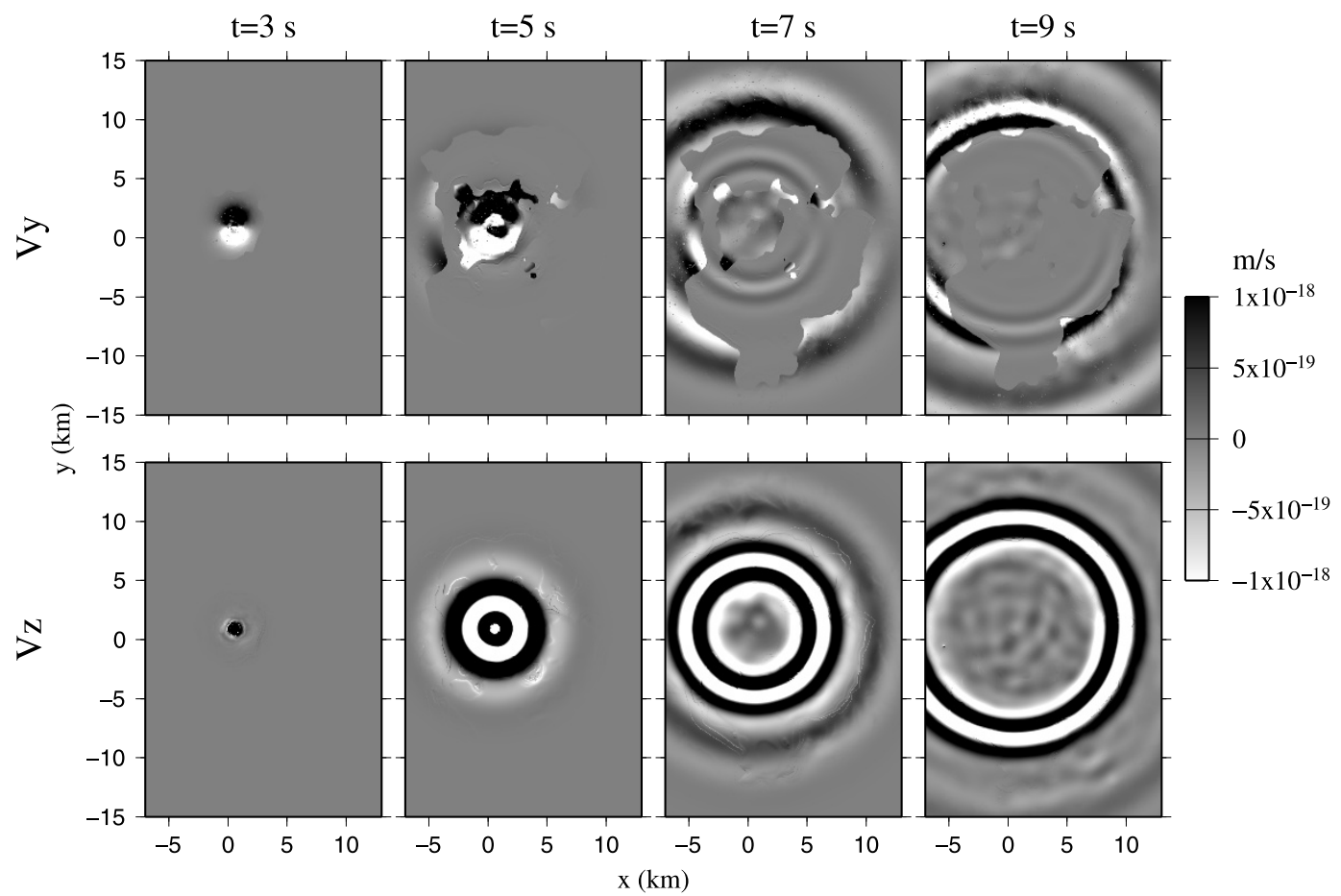

Fig. 3. Snapshots of synthetic velocities $V_{y}$ (upper panels) and $V_{z}$ (lower panels) at half grids below the land and water surfaces at $t=3,5,7$, and $9 \mathrm{~s}$ calculated with the water model using $T_{p}=2 \mathrm{~s}$ and a source at $220 \mathrm{~m}$ below sea level.

shots of the $y$-component velocity $V_{y}$ (upper panels), indicating that the wave amplitudes are highly weakened near the water surfaces. In TL, there is a ring-like pattern corresponding to wave packets, indicating that the wave energies are partly transmitted to the water domains. When we look at the snapshots of the vertical velocity $V_{z}$ (lower panels), the wave amplitudes in the lake regions are not weakened. This difference among the components may be attributed to the boundary conditions. For the normal component, the velocity is continuous on the solid-water interfaces and is not zero on the water surfaces. On the other hand, the tangential velocity components are not continuous on the solid-water interfaces and become zero on the water surfaces. The latter condition arises from the equation of motion $\rho \partial V_{y} / \partial t=-\partial p / \partial y$ with the pressure $p=0$ on the water surfaces. We note that the snapshots of $V_{y}$ in Fig. 3, in the lake region, are not zero because they are at half grids below the water surfaces in the staggered grid scheme.

In the following, we used the black dotted rectangle in Fig. 1(b) as the horizontal range of the computational domain to save computational time and memory. To check if this domain was wide enough, we calculated waveforms using the entire domain of Fig. 1(b) for the water model with $T_{p}=2 \mathrm{~s}$ and a source depth of $220 \mathrm{~m}$. Figure 4 compares the waveforms obtained using these two domains. We can see no distinct difference at the main portions of the waveforms and only a minor difference in the later portions. We quantify the waveform differences obtained using the two domains through misfits, defined as

$$
\delta_{\mathrm{w}}^{\prime}=\sqrt{\frac{\sum_{i=x, y, z} \sum_{k=0}^{N-1}\left[V_{i}^{\mathrm{w}}(k \Delta t)-V_{i}^{\mathrm{w} \prime}(k \Delta t)\right]^{2}}{\sum_{i=x, y, z} \sum_{k=0}^{N-1}\left[V_{i}^{\mathrm{w} \prime}(k \Delta t)\right]^{2}}},
$$

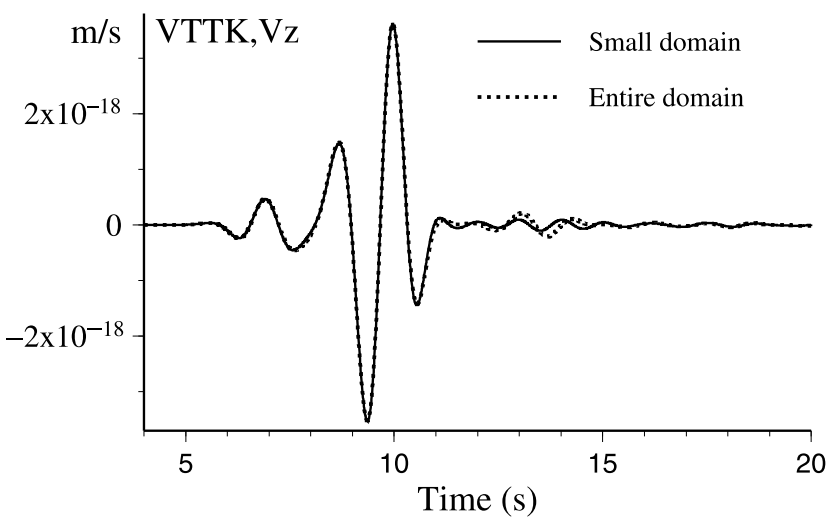

Fig. 4. Synthetic vertical velocities at station VTTK calculated with the water model using $T_{p}=2 \mathrm{~s}$ and a source at $220 \mathrm{~m}$ below sea level. Solid and dotted lines indicate the waveforms calculated using the computational domains represented by the black dotted rectangle in Fig. 1(b) and the entire domain of Fig. 1(b), respectively.

where $V_{i}^{\mathrm{w}}$ and $V_{i}^{\mathrm{w} \prime}$ denote synthetic velocity components in the $i$-direction at a station calculated with the water model using the small and entire domains, respectively. We calculated the misfit value at each station shown in Fig. 1(b). The largest misfit was 0.13 at the northernmost virtual station (VSN) and the second largest was 0.082 . Both of these were small enough to have a negligible effect on the following conclusions, which justify the use of the small domain in the following calculations.

Figure 5 shows the synthetic seismograms at near- and far-field stations, VTDK and VTTK (Fig. 1), calculated for the three models for $T_{p}=1 \mathrm{~s}$ and a source depth of 220 m. At VTTK (Fig. 5(b)), clear phase shifts among the three models are evident in the Rayleigh wave portion, whereas 

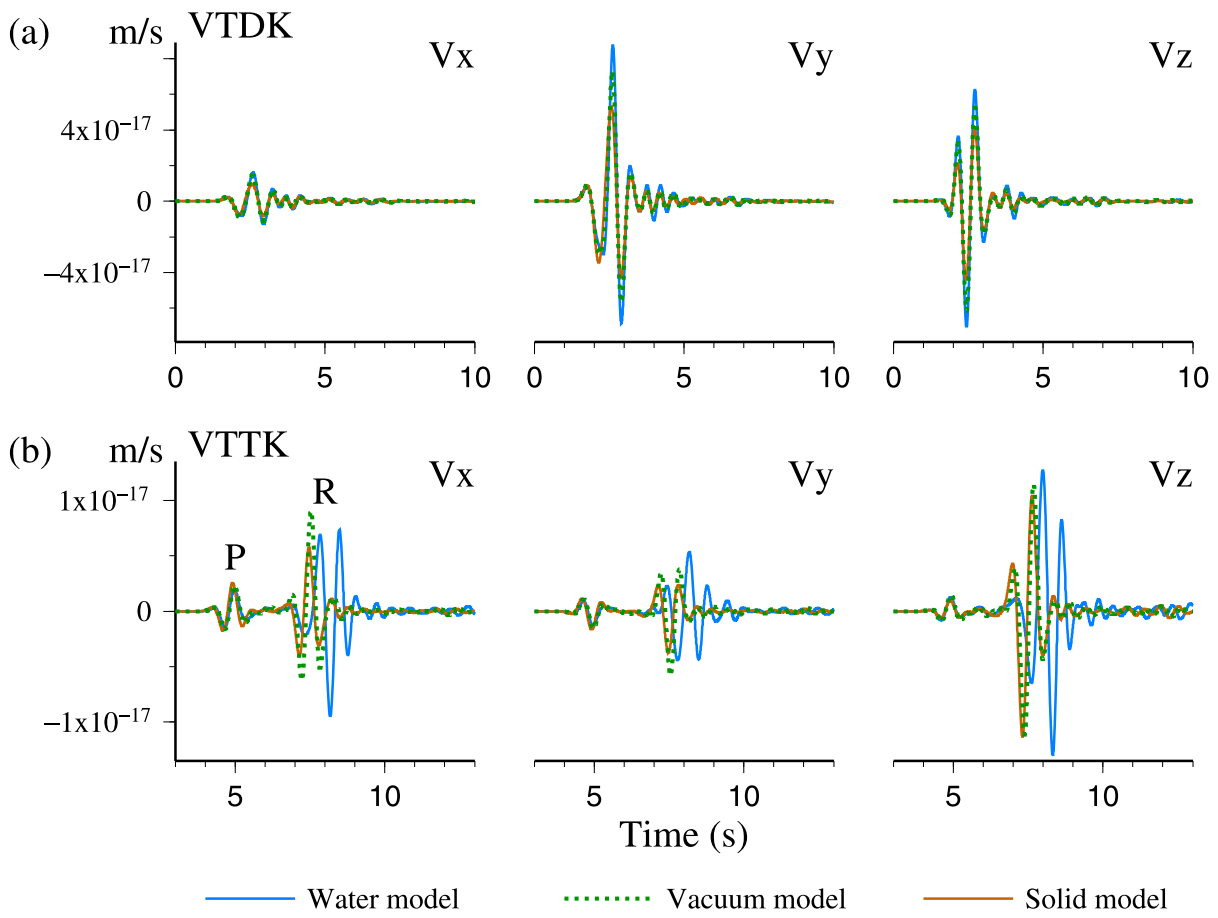

Fig. 5. Synthetic seismograms at stations VTDK and VTTK calculated with the water (blue lines), vacuum (green dotted lines), and solid (brown lines) models using $T_{p}=1 \mathrm{~s}$ and a source at $220 \mathrm{~m}$ below sea level. 'P' and 'R' denote $P$ and Rayleigh waves, respectively.
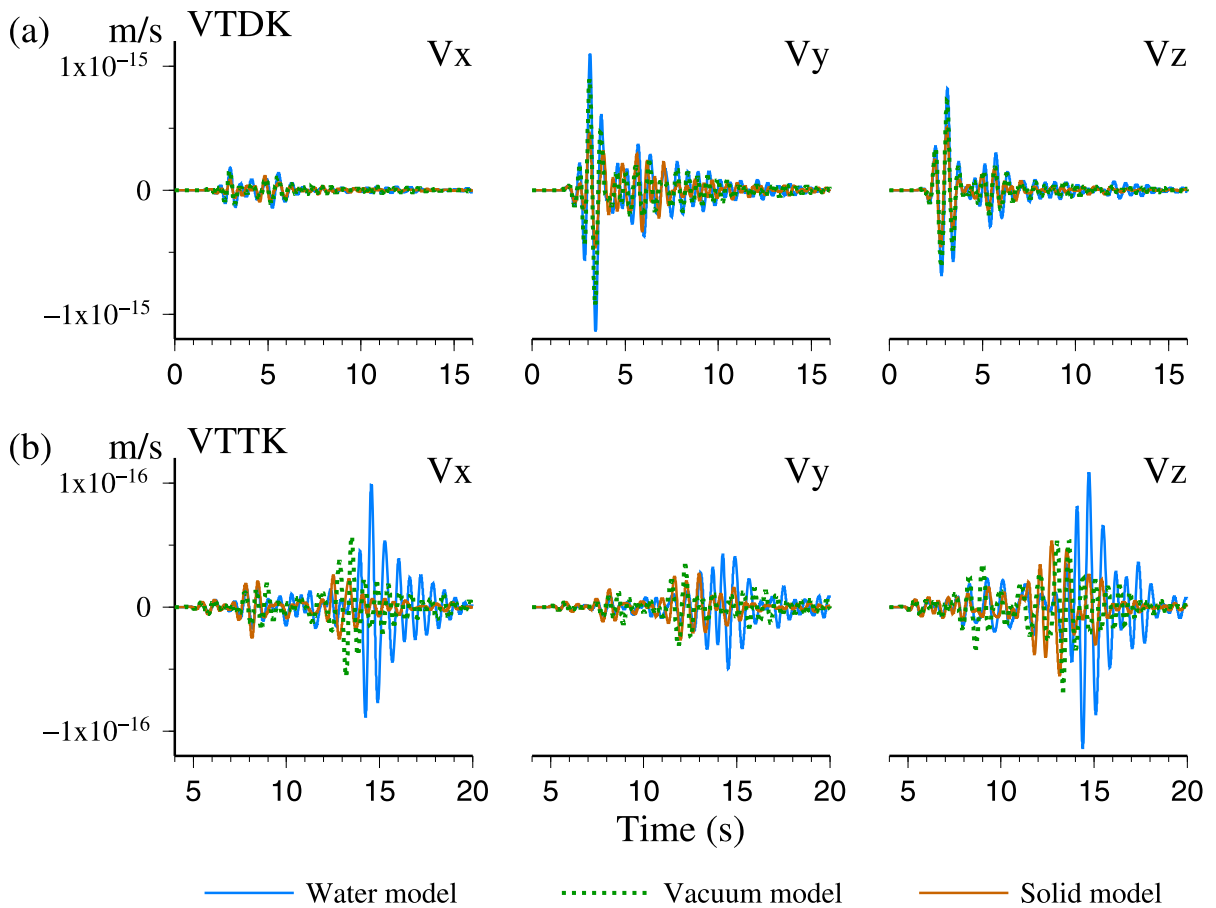

Fig. 6. Synthetic seismograms at stations VTDK and VTTK calculated with the water (blue lines), vacuum (green dotted lines), and solid (brown lines) models using $T_{p}=1 \mathrm{~s}$ and a source at $220 \mathrm{~m}$ below sea level. A layered structure was used for the solid domain.

the waveform differences among the three models are small in the $P$-wave portion. At VTDK (Fig. 5(a)), differences in amplitude among the three models appear for the entire duration of the main wave packet, but the phase shifts are not observed.

We further performed simulations, in which we used a layered structure for the solid domain. The structure contained three layers: layer 1 from the surface to $500 \mathrm{~m}$ be- low sea level with $V_{p}=2000 \mathrm{~m} / \mathrm{s}$, layer 2 ranging from 500 to $2000 \mathrm{~m}$ below sea level with $V_{p}=3100 \mathrm{~m} / \mathrm{s}$, and a half space below layer 2 with $V_{p}=4300 \mathrm{~m} / \mathrm{s}$. We used $V_{s}=V_{p} / \sqrt{3}$ and $\rho=1700+0.2 V_{p}$ (Gardner et al., 1974). We used the same source as used in the previous test. Figure 6(b) compares synthetic waveforms at VTTK calculated for the three models with the layered solid structure. A large amplification, and a phase delay of the Rayleigh wave, is 

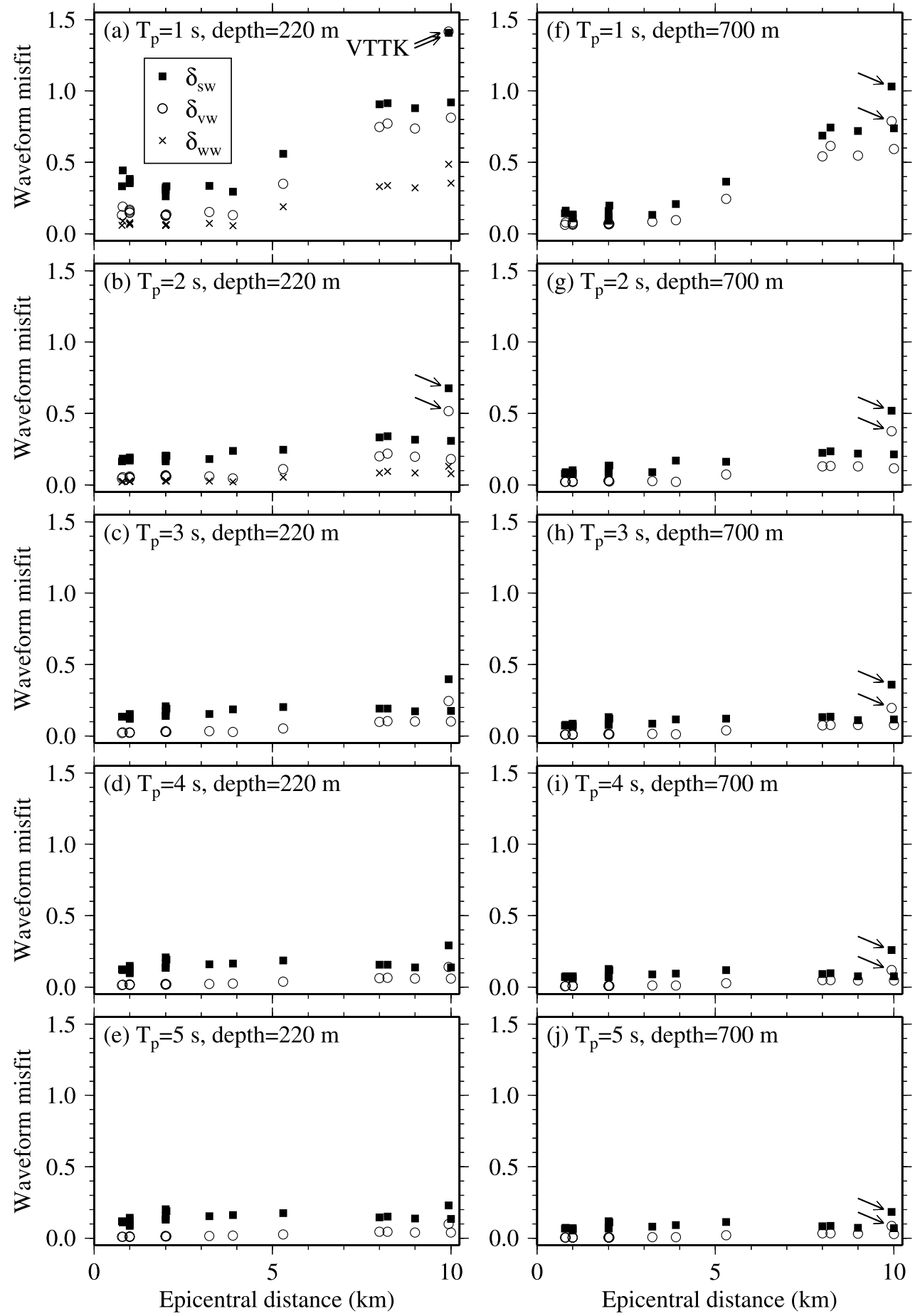

Fig. 7. Plots of waveform misfits for various models $\left(\delta_{\mathrm{sw}}, \delta_{\mathrm{vw}}\right.$, and $\delta_{\mathrm{ww}}$, Eqs. (5)-(7)), as functions of the epicentral distance for various source durations $T_{p}$ and depths below sea level. We used $T_{s}=2 T_{p}$ in Eq. (2). Arrows indicate the misfits at VTTK.

prominent in the water model. This result indicates that the water domains have a large effect on the Rayleigh wave portion at far-field stations regardless of a homogeneous, or a layered, solid structure. At VTDK (Fig. 6(a)), we can see amplitude differences among the three models in the entire duration of the main wave packet but the phase shifts are small. These characteristics at near-field stations are consistent with the simulation results obtained using the homogeneous solid structure (Fig. 5(a)). Given these conclusions, we hereafter show the results obtained using the homogeneous solid structure for simplicity.
From the individual waveform traces, we calculated the waveform misfits among the three models, defined as

$$
\begin{gathered}
\delta_{\mathrm{sw}}=\sqrt{\frac{\sum_{i=x, y, z} \sum_{k=0}^{N-1}\left[V_{i}^{\mathrm{s}}(k \Delta t)-V_{i}^{\mathrm{w}}(k \Delta t)\right]^{2}}{\sum_{i=x, y, z} \sum_{k=0}^{N-1}\left[V_{i}^{\mathrm{w}}(k \Delta t)\right]^{2}}}, \\
\delta_{\mathrm{vw}}=\sqrt{\frac{\sum_{i=x, y, z} \sum_{k=0}^{N-1}\left[V_{i}^{\mathrm{v}}(k \Delta t)-V_{i}^{\mathrm{w}}(k \Delta t)\right]^{2}}{\sum_{i=x, y, z} \sum_{k=0}^{N-1}\left[V_{i}^{\mathrm{w}}(k \Delta t)\right]^{2}}},
\end{gathered}
$$

where $V_{i}^{\mathrm{v}}$ and $V_{i}^{\mathrm{s}}$ denote synthetic velocity components in 
the $i$-direction calculated with the vacuum and solid models, respectively. We calculated the mis ts between the solid and water models $\left(\delta_{\mathrm{sw}}\right)$ and between the vacuum and water models $\left(\delta_{\mathrm{vw}}\right)$ for ten combinations of source duration and depth, which we plotted as functions of epicentral distance (Fig. 7).

For all ten cases, the mis ts between the vacuum and water models $\left(\delta_{\mathrm{vw}}\right)$ are generally smaller than those between the solid and water models $\left(\delta_{\mathrm{sw}}\right)$, indicating that the vacuum model provides a better approximation of the water domain than the solid model. The mis ts $\delta_{\mathrm{sw}}$ and $\delta_{\mathrm{vw}}$ for the shallower source (Figs. 7(a)-7(e)) are larger than those for the deeper source (Figs. 7(f)-7(j)). The maximum values of $\delta_{\mathrm{sw}}$ for $T_{p}=1$ and $2 \mathrm{~s}$ and source depth of $220 \mathrm{~m}$ are 1.41 and 0.68 , respectively (Figs. 7 (a) and 7(b)), indicating that the water domains markedly affect the seismic waveforms when the period is shorter than $2 \mathrm{~s}$. The mis$\mathrm{t} \delta_{\text {sw }}$ gradually decreases with increasing source duration $T_{p}$ (Figs. 7(c) and 7(d)) and becomes smaller than 0.23 for $T_{p}=5 \mathrm{~s}$ (Fig. 7(e)). Similar characteristics are also visible for mis $\mathrm{t} \delta_{\mathrm{vw}}$ (Figs. 7(a)-7(e)) and for a source depth of $700 \mathrm{~m}$ (Figs. 7(f)-7(j)). We also conducted calculations for $T_{p}=10 \mathrm{~s}$, which resulted in similar mis $\mathrm{t}$ values to those for $T_{p}=5 \mathrm{~s}$. These results consistently indicate that the effects of the water domains are relatively small when the period is longer than $5 \mathrm{~s}$.

For $T_{p}=1$ and $2 \mathrm{~s}$ for stations not on the island (epicentral distance $>5 \mathrm{~km}$ ), the waveform mis ts $\delta_{\mathrm{sw}}$ and $\delta_{\mathrm{vw}}$ increase as the epicentral distance increases (Figs. 7(a), 7(b), $7(\mathrm{f})$, and $7(\mathrm{~g}))$. These increasing trends suggest that the waveform mis ts among the three models represent the effect of TL. Figure 7 also indicates that the mis ts at VTTK are larger than those at the virtual station VSN located at a similar epicentral distance $(10 \mathrm{~km})$. The TL portion on the ray path between the source and VTTK was longer than that between the source and VSN (Fig. 1), which caused the larger mis $\mathrm{t}$ values at VTTK. On the other hand, for $T_{p}=1 \mathrm{~s}$ and a source depth of $220 \mathrm{~m}$ (Fig. 7(a)), the mis-

ts at the closest station are relatively large and decrease as the epicentral distance increases within the island (epicentral distance $\leq 4 \mathrm{~km}$ ), which we consider to be the effect of MCL. These results indicate that the water domains have both near- and far- eld effects.

In Section 2, we evaluated the waveform mis ts $\delta_{\mathrm{FD}}$ (Eq. (3)) between the FDM and DWM for water-layer thicknesses of 40 and $160 \mathrm{~m}$ (Fig. 2), which correspond to typical depths of MCL and TL, respectively. In the evaluation, we used a source with $T_{p}=1 \mathrm{~s}$ at $220 \mathrm{~m}$ depth, which were the same as those used in Fig. 7(a). Hence, the mis ts $\delta_{\mathrm{FD}}$ may be used as rough estimations of the FDM calculation errors in Fig. 7(a). At an epicentral distance of $1 \mathrm{~km}, \delta_{\mathrm{FD}}$ for a water-layer thickness of $40 \mathrm{~m}$ (0.03, Fig. 2(a)) may be appropriate for the rough error estimation because the waveforms are mainly affected by MCL. This error (0.03) is small compared to the waveform mis ts $\delta_{\mathrm{sw}}$ and $\delta_{\mathrm{vw}}$ at the same epicentral distance $(0.15-0.38$, Fig. 7(a)). At an epicentral distance of $10 \mathrm{~km}$, the waveforms are mainly affected by TL so that $\delta_{\mathrm{FD}}$ for a water-layer thickness of 160 $\mathrm{m}(0.36$, Fig. 2(d)) may be appropriate for the error estimation. This relatively large error (0.36) is mainly attributed to the Rayleigh wave portion of the waveform propagating through the water domain (Fig. 2(d)), which may be caused by our use of only a few vertical grid cells in the water domain. However, the mis ts $\delta_{\mathrm{sw}}$ and $\delta_{\mathrm{vw}}$ at the same epicentral distance (0.81-1.42, Fig. 7(a)) are 2-4 times larger than this error (0.36). Therefore, our interpretation of the near- and far- eld effects may not be seriously affected by the FDM calculation errors.

The synthetic waveforms may be affected by our treatment of the solid-water boundary. To check this point, we also calculated synthetic waveforms $\left(V_{i}^{\text {wd }}\right)$ using the density of water as the density at the solid-water boundary, as a natural extension of the work of Ohminato and Chouet (1997). We note that this treatment is different from that described in Section 2 of this study. We compared the waveforms $V_{i}^{\text {wd }}$ with $V_{i}^{\text {w }}$ through mis ts

$$
\delta_{\mathrm{ww}}=\sqrt{\frac{\sum_{i=x, y, z} \sum_{k=0}^{N-1}\left[V_{i}^{\mathrm{wd}}(k \Delta t)-V_{i}^{\mathrm{w}}(k \Delta t)\right]^{2}}{\sum_{i=x, y, z} \sum_{k=0}^{N-1}\left[V_{i}^{\mathrm{w}}(k \Delta t)\right]^{2}}} .
$$

Crosses in Figs. 7(a) and 7(b) represent $\delta_{\mathrm{ww}}$ for $T_{p}=1$ and $2 \mathrm{~s}$, respectively. The maximum value of $\delta_{\mathrm{ww}}$ is 0.49 for $T_{p}=1 \mathrm{~s}$, indicating that the synthetic seismograms are affected by the treatment of the solid-water boundary.

\section{Waveform Inversion of Synthetic Data 4.1 Inversion procedure}

We conducted waveform inversion using synthetic waveforms to examine how the water domains affect inversion solutions. Synthetic observed seismograms at the real station locations were calculated using the water model, and the three models (water, vacuum, and solid models) were used for calculations of Green's functions, in which we used the homogeneous solid structure. We conducted a least-squares inversion for six moment tensor time functions based on the method of Auger et al. (2006), in which waveform inversion is performed in the frequency domain to reduce the computational time and computer memory requirements.

We synthesized observed seismograms assuming a point vertical tensile crack source with an N-S opening direction. We set the crack source beneath MCL at $220 \mathrm{~m}$ below sea level, under the red solid circle in Fig. 1. We used a Ricker wavelet characterized by $T_{p}=2 \mathrm{~s}$ and $T_{s}=4 \mathrm{~s}$ as the source time function. Source depths of 100-300 m have been commonly estimated for LP and VLP events at various volcanoes (e.g., Hidayat et al., 2002; Chouet et al., 2003; Nakano et al., 2003; Maeda and Takeo, 2011). Because the Eurasian plate subducts eastward (Galgana et al., 2007; Ku et al., 2009), a tensile crack with an N-S opening direction is a plausible source mechanism at Taal volcano.

Because the source location was xed to the input location in our waveform inversion, we calculated Green's functions excited at the input source location only. We used the same computational domain, grid interval, and time step as those we used in the forward synthetic tests. For our calculation of Green's functions, we used a pulsive source time function of $1 \mathrm{~s}$ duration, which was deconvolved through the frequency-domain inversion process. We calculated synthetic observed seismograms and Green's functions with 
(a) Water model

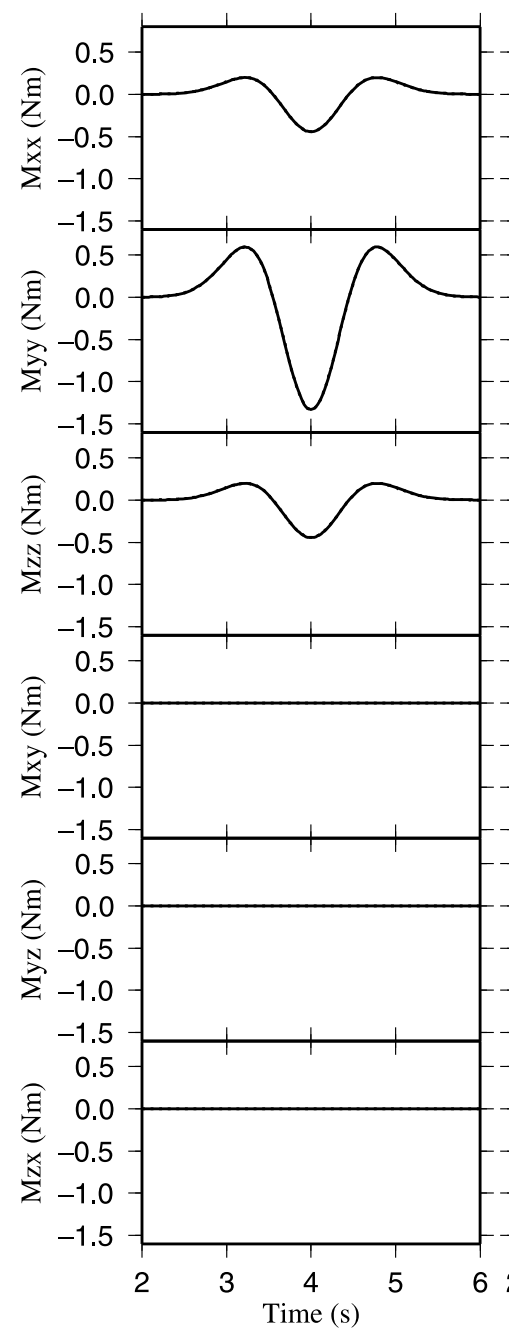

(b) Vacuum model

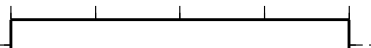

(c) Solid model

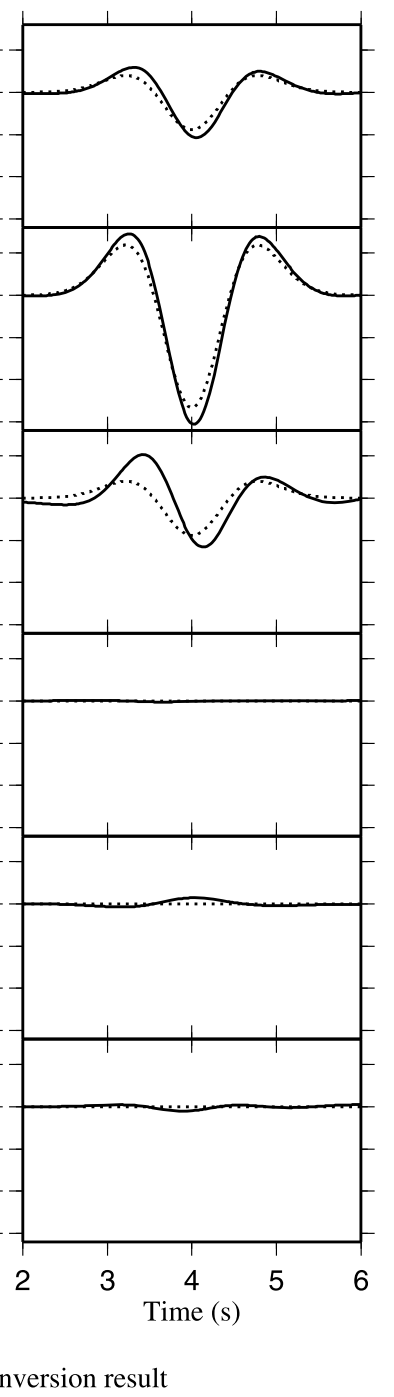

Fig. 8. Source time functions of six moment tensor components of the waveform inversion solutions, which were determined using synthetic seismograms calculated with the water model and Green's functions calculated with (a) water, (b) vacuum, and (c) solid models.

Table 1. List of station configurations for synthetic inversions conducted in this study. Stations used in inversions are indicated by asterisks $(*)$.

\begin{tabular}{ccccccc}
\hline & \multicolumn{7}{c}{ Station network } \\
\cline { 2 - 7 } & VTDK & VTCT & VTBM & VTNP & VTBC & VTTK \\
\hline $\mathrm{A}$ & $*$ & $*$ & $*$ & $*$ & $*$ & \\
\hline $\mathrm{B}$ & & $*$ & $*$ & $*$ & $*$ & \\
\hline $\mathrm{C}$ & & & $*$ & $*$ & $*$ & $*$ \\
\hline
\end{tabular}

a total duration of $20 \mathrm{~s}$, and decimated them to a sampling interval of $0.02 \mathrm{~s}$. We used three different station configurations in our waveform inversion (see Table 1) to evaluate the dependence of the inversion solutions on station configuration.

\subsection{Inversion results}

We first used station configuration A (Table 1) in our inversion. Figure 8 shows source time functions of six moment tensor components estimated by our waveform inversion using Green's functions calculated with the three models. Figures 9 and 10 describe the principal axes corresponding to these three inversion solutions. Here, $M_{1}$, $M_{2}$, and $M_{3}$ indicate the largest, intermediate, and smallest eigenvalues for the principal axes, respectively, and $\alpha_{1}$ indicates the angle between $M_{1}$ and the $y$-axis. If the inversion solution is perfect, $M_{2} / M_{1}=M_{3} / M_{1}=1 / 3$ and $\alpha_{1}=0$ in Fig. 9, and the tensile directions shown in Fig. 10 are oriented in the N-S direction. Since we defined the eigenvalues to satisfy $\left|M_{1}(t)\right|>\left|M_{2}(t)\right|>\left|M_{3}(t)\right|$ at each time $t$, jumps of the eigenvalues occurred at times when the order of the eigenvalues changed (Fig. 9), although the moment tensor solutions were continuous with time (Fig. 8). When the water model was used to calculate Green's functions, the inversion solutions showed almost perfect recoveries, as expected (Figs. 8(a), 9(a), and 10(a)). The inversion solutions obtained using the vacuum and solid models show de- 
(a) Water model

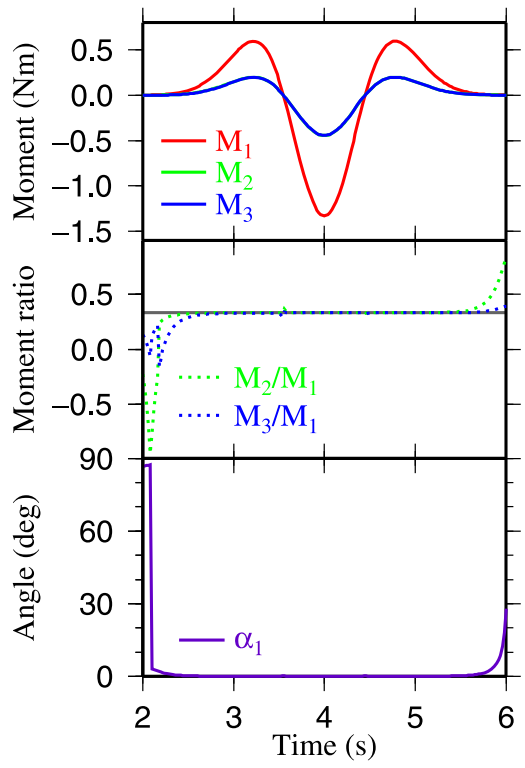

(b) Vacuum model

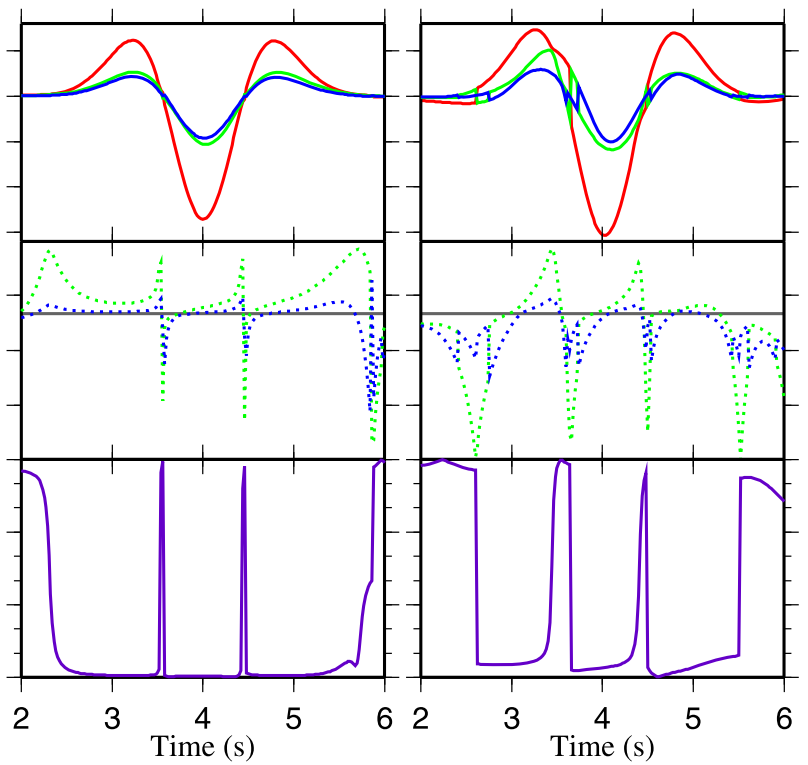

Fig. 9. Plots of the principal axes as functions of time, estimated from the source time functions of the six moment tensor components shown in Fig. 8. $M_{1}(t), M_{2}(t)$, and $M_{3}(t)$ indicate the largest, intermediate, and smallest eigenvalues for the principal axes, respectively, and $\alpha_{1}(t)$ indicates the angle between the $y$-axis and the principal axis corresponding to $M_{1}$. Gray horizontal lines in the middle panels indicate the input crack source for which $M_{2} / M_{1}=M_{3} / M_{1}=1 / 3$.

(a) Water model

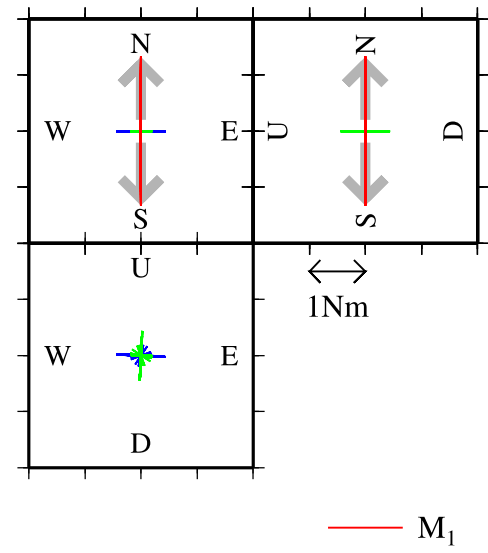

(b) Vacuum model

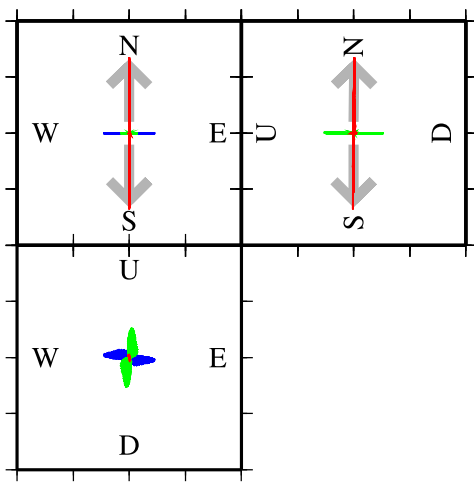

$\mathrm{M}_{2}$ (c) Solid model

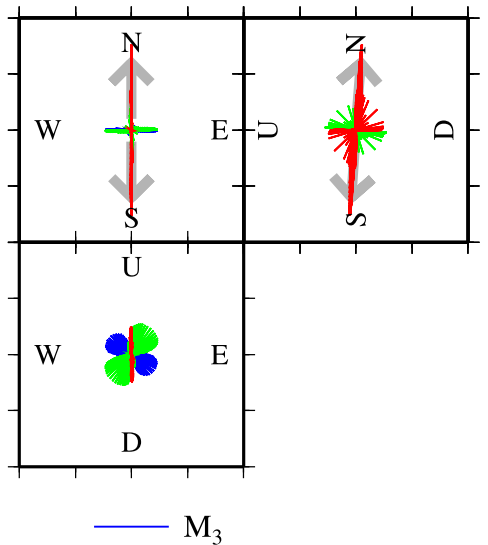

Fig. 10. Plots of eigenvectors for the principal axes in space, estimated from the source time functions of the six moment tensor components shown in Fig. 8. Red, green, and blue lines indicate eigenvectors corresponding to $M_{1}, M_{2}$, and $M_{3}$, respectively. The length of each line is proportional to the eigenvalue of the principal axis. Gray arrows in each panel indicate the direction of the principal axis corresponding to the maximum value of $\left|M_{1}(t)\right|$.

viations from the input source. In particular, the deviations of $M_{z z}$ (Figs. 8(b) and 8(c)) and $M_{2}$ (Figs. 9(b) and 9(c)) are large for both of these models. In the inversion result obtained using the solid model, the crack deviates from the vertical (gray arrows in Fig. 10(c)). The deviations of the solutions obtained using the solid model are clearly larger than those obtained using the vacuum model.

We calculated errors of the inversion results, defined as

$$
E_{i}=\sqrt{\frac{\sum_{k=k_{1}}^{k_{2}}\left[M_{i}^{\text {inv }}(k \Delta t)-M_{i}^{\text {input }}(k \Delta t)\right]^{2}}{\sum_{k=k_{1}}^{k_{2}}\left[M_{i}^{\text {input }}(k \Delta t)\right]^{2}}},
$$

where $i=1,2,3$, and the superscripts 'inv' and 'input' indicate eigenvalues for the inversion results and the input source, respectively. Here, $k_{1} \Delta t$ and $k_{2} \Delta t$ define the time window shown in Figs. 8 and 9 (i.e. $k_{1} \Delta t=2 \mathrm{~s}$ and $\left.k_{2} \Delta t=6 \mathrm{~s}\right)$. We compared $E_{i}$ obtained from the three models with various station configurations (Fig. 11). For the water model, the errors are smaller than 0.04 for all parameters and station configurations. The errors for the solid model are generally larger than those for the vacuum model. For example, the errors in $M_{2}\left(E_{2}\right)$ using station configuration A are 0.30 and 0.77 for the vacuum and solid models, respectively.

It may be expected that the errors decrease if we use data closer to the source. This tendency generally holds for inversion results obtained using the vacuum model (Fig. 11), in which the inversion using station configuration A including data at station VTDK (Table 1) provided the smallest errors for individual parameters among the three station con- 


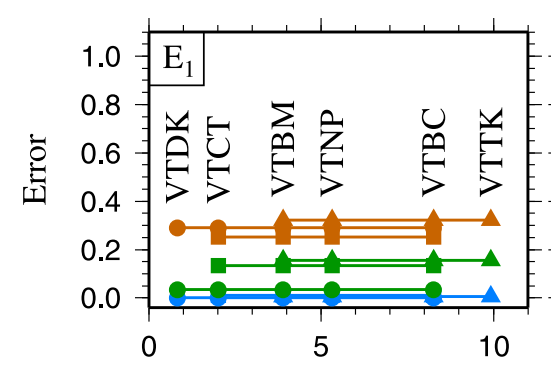

Water model
Station Configuration:

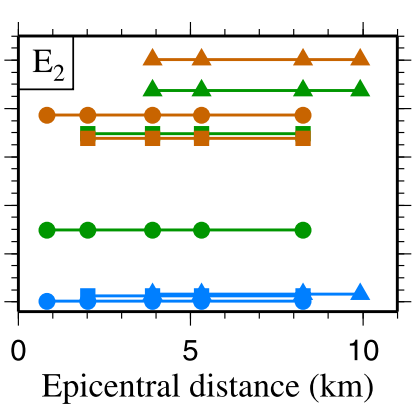

- Vacuum model

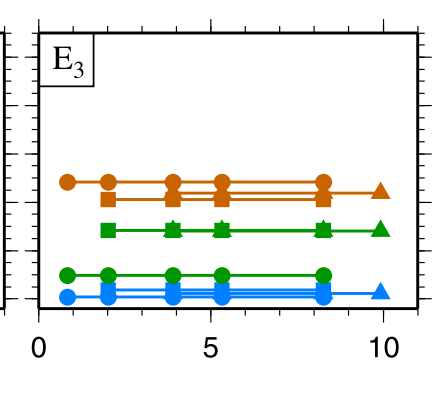

Solid model

$$
\bullet \bullet \mathrm{A} \quad \square \square \mathrm{B} \quad \Delta \Delta \mathrm{C}
$$

Fig. 11. Plots of the errors of the waveform inversion solutions defined by Eq. (8). Inversions were conducted using synthetic observed seismograms calculated with the water model and Green's functions calculated with the water (blue lines), vacuum (green lines), and solid (brown lines) models. The range of the lines and the circle, square, and triangle symbols indicate the station configurations (Table 1) used in the inversions.

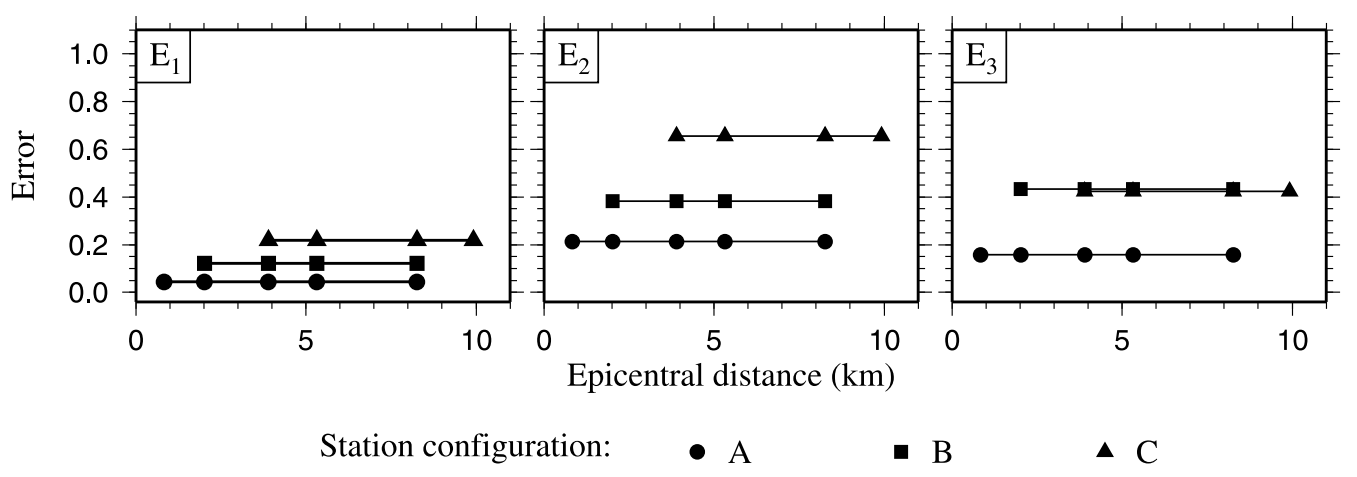

Fig. 12. Plots of the errors of the waveform inversion solutions defined by Eq. (8). Inversions were conducted using synthetic observed seismograms calculated with a composite model and Green's functions calculated with the solid model, for various station configurations listed in Table 1. The composite model consists of MCL and TL filled with solid and water, respectively.

figurations. However, in the inversion results obtained using the solid model, the use of data at VTDK increased the errors (Fig. 11). We speculate that this represents the effect of MCL. To check this assumption, we performed an additional inversion test. We used a composite model, in which MCL and TL were filled with solid and water, respectively, for the calculation of synthetic observed seismograms. We performed waveform inversion of these seismograms using Green's functions calculated with the solid model. In this way, we eliminated the effect of the MCL structure. The errors for this case (Fig. 12) indicate that station configuration A provided the smallest errors in the individual parameters. We can thus conclude that the large errors in the inversion solutions for station configuration A, shown by the brown lines in Fig. 11, are caused mainly by the replacement of water by the solid medium at MCL.

\section{Discussion}

Our forward waveform simulation revealed that water domains have a considerable effect on seismic waveforms when the period is shorter than $2 \mathrm{~s}$ (Fig. 7), whereas their effects on waveforms are small when the source duration is longer than $5 \mathrm{~s}$. Our simulation also showed that the water model is better approximated by a vacuum model than a solid model.

The seismograms at distant stations beyond the Volcano
Island are dominantly affected by the outer lake (TL). Synthetic seismograms at station VTTK (Fig. 5(b)) display relatively large waveform differences among the three models for the Rayleigh wave portions of the waveforms. At station VTTK, amplitude differences among the models are minor, but a phase delay of the Rayleigh wave is prominent in the water model (Fig. 5(b)). This phase delay may be caused by dispersion of the Rayleigh wave propagating through the water domain in TL. Although Fig. 5(b) was obtained using the homogeneous structure for the solid domain, our simulation using the layered structure for the solid domain (Fig. 6) also showed that the water domains affect the Rayleigh wave portions of the waveforms at farfield stations.

Hatayama (2004) performed 2D waveform simulations to examine the effect of an oceanic layer on waveforms with periods of $0.18-6$ s observed at an epicentral distance of 60 $\mathrm{km}$ using both homogeneous and layered solid structures. Petukhin et al. (2010) performed 3D waveform simulations for periods of a few seconds, and epicentral distances of $\sim 20 \mathrm{~km}$, to examine the effect of an oceanic layer using a 3D layered solid structure of the Kinki region, Japan. Both studies concluded that (1) the oceanic layer affects the Rayleigh wave portion of the waveforms at far-field stations, and (2) the effect of the oceanic layer becomes minor with increasing source depth. Hatayama (2004) also 
concluded that the water layer is better approximated by vacuum than solid. These conclusions are consistent with our results for the far- eld waveforms (Figs. 5(b), 6(b), and 7).

On the other hand, we showed that seismograms at stations close to the source are affected by MCL (Fig. 7). At these stations, body and surface waves arrive almost simultaneously because the hypocentral distances are small. The waveform differences among the three models at station VTDK (Fig. 5(a)) are visible for the entire duration of the main wave packet. At station VTDK, the seismic amplitudes are smallest for the solid model; those of the water and vacuum models are almost the same. The waveform mists at stations on the island for $T_{p}=1 \mathrm{~s}$ and source depth $220 \mathrm{~m}$ tend to be larger at nearer stations (Fig. 7(a)). On the other hand, when the source is deeper $(700 \mathrm{~m})$, this tendency is not observed (Fig. 7(f)). Thus, we consider that the waveform mis ts at stations on the island re ect the neareld effect of MCL. Our simulations thus demonstrate that water domains have both near- and far- eld effects.

Although the waveforms calculated by the FDM code may have some errors (Fig. 2), the waveform mis ts among the three models (Fig. 7) are larger than the estimated errors at near- and far- eld stations (Section 3) so that our interpretation of the near-and far- eld effects may not be seriously affected by FDM calculation errors.

Our inversion tests support our interpretation of the simulations. The inversion solutions obtained using the solid model were overestimated (Fig. 8(c)), which can be explained by the smallest near- eld seismic amplitudes in the solid model. On the other hand, the inversion test using the vacuum model provided solutions similar to the input source (Fig. 8(b)). For the solid model, the use of data from station VTDK worsened the errors in the inversion results (Fig. 11), which is explained by the near- eld effect. When we eliminated the structural effect of MCL, however, the errors in the inversion results were generally smaller when using data from stations closer to the source (Fig. 12). This is explained by the far- eld effect at distant stations, where the waveform differences increase due to the dispersion of the Rayleigh wave.

Chouet et al. (2003) compared synthetic seismograms at the Stromboli volcano, Italy, with and without a sea layer, and concluded that the effect of the sea layer was minor. Their results are consistent with ours, because (1) they used water and vacuum models, not a solid model, for their comparison, (2) the dominant periods of the waveforms in their study were around $5 \mathrm{~s}$, and (3) all of the stations they used were on an island surrounded by sea.

\section{Conclusions}

We examined the effects of water domains on seismic wave elds at Taal volcano, Philippines, a volcano with both crater and caldera lakes. We conducted forward waveform simulation and synthetic waveform inversion using the 3D nite-difference method with and without water lakes. Our results suggest that the water domains have both near- and far- eld effects on seismic wave elds. The near- eld effect is related to source excitation affected by a water domain, whereas the far- eld effect is caused by the dispersion of
Rayleigh waves propagating through a water domain. Our study also showed that the replacement of water by a vacuum, rather than a solid medium, provides a better approximation of a water domain. A water domain has a marked effect on seismic waveforms and the results of waveform inversion when the waveform period is shorter than $2 \mathrm{~s}$. If this is the case, a water domain must be properly accounted for, even if the seismic stations lie in the near eld. We also showed that the effect of a water domain is small for waveform periods longer than $5 \mathrm{~s}$.

Acknowledgments. We thank Taro Okamoto for providing numerical data to check the FDM calculations. The DEM of Taal volcano that we used was provided by the Philippine Institute of Volcanology and Seismology (PHIVOLCS). This study was nancially supported by the Japan Science and Technology Agency (JST) and the Japan International Cooperation Agency (JICA). Comments from two anonymous reviewers helped to improve the manuscript.

\section{References}

Auger, E., L. D’Auria, M. Martini, B. Chouet, and P. Dawson, Realtime monitoring and massive inversion of source parameters of very long period seismic signals: An application to Stromboli Volcano, Italy, Geophys. Res. Lett., 33, L04301, doi:10.1029/2005GL024703, 2006.

Chew, W. C. and Q. H. Liu, Perfectly matched layers for elastodynamics: a new absorbing boundary condition, J. Comput. Acoust., 4, 341-359, 1996.

Chouet, B., P. Dawson, T. Ohminato, M. Martini, G. Saccorotti, F. Giudicepietro, G. De Luca, G. Milana, and R. Scarpa, Source mechanisms of explosions at Stromboli Volcano, Italy, determined from momenttensor inversions of very-long-period data, J. Geophys. Res., 108, 2019, doi:10.1029/2002JB001919, 2003.

Delmelle, P., M. Kusakabe, A. Bernard, T. Fischer, S. de Brouwer, and E. del Mundo, Geochemical and isotopic evidence for seawater contamination of the hydrothermal system of Taal Volcano, Luzon, the Philippines, Bull. Volcanol., 59, 562-576, 1998.

Festa, G. and S. Nielsen, PML absorbing boundaries, Bull. Seismol. Soc. Am., 93, 891-903, doi:10.1785/0120020098, 2003.

Galgana, G., M. Hamburger, R. McCaffrey, E. Corpuz, and Q. Chen, Analysis of crustal deformation in Luzon, Philippines using geodetic observations and earthquake focal mechanisms, Tectonophysics, 432, 63-87, doi:10.1016/j.tecto.2006.12.001, 2007.

Gardner, G. H., L. W. Gardner, and A. R. Gregory, Formation velocity and density-The diagnostic basics for stratigraphic traps, Geophysics, 39, 770-780, doi:10.1190/1.1440465, 1974.

Hatayama, K., Theoretical evaluation of effects of sea on seismic ground motion, in Proceedings of the 13th World Conference on Earthquake Engineering, Vancouver, Canada, 2004, paper 3229, 2004.

Hidayat, D., B. Chouet, B. Voight, P. Dawson, and A. Ratdomopurbo, Source mechanism of very-long-period signals accompanying dome growth activity at Merapi volcano, Indonesia, Geophys. Res. Lett., 29, 2118, doi:10.1029/2002GL015013, 2002.

Kobayashi, T., T. Ohminato, Y. Ida, and E. Fujita, Very long period seismic signals observed before the caldera formation with the 2000 Miyake-jima volcanic activity, Japan, J. Geophys. Res., 114, B02211, doi:10.1029/2007JB005557, 2009.

Ku, Y.-P., C.-H. Chen, S.-R. Song, Y. Iizuka, and J.-S. Shen, A 2 Ma record of explosive volcanism in southwestern Luzon: Implications for the timing of subducted slab steepening, Geochem. Geophys. Geosyst., 10, Q06017, doi:10.1029/2009GC002486, 2009.

Kumagai, H., T. Ohminato, M. Nakano, M. Ooi, A. Kubo, H. Inoue, and J. Oikawa, Very-long-period seismic signals and caldera formation at Miyake Island, Japan, Science, 293, 687-690, doi:10.1126/science.1062136, 2001.

Kumagai, H., K. Miyakawa, H. Negishi, H. Inoue, K. Obara, and D. Suetsugu, Magmatic dike resonances inferred from very-long-period seismic signals, Science, 299, 2058-2061, doi:10.1126/science.1081195, 2003.

Legrand, D., S. Kaneshima, and H. Kawakatsu, Moment tensor analysis of near- eld broadband waveforms observed at Aso Volcano, Japan, $J$. Volcanol. Geotherm. Res., 101, 155-169, 2000.

Maeda, Y. and M. Takeo, Very-long-period pulses at Asama volcano, cen- 
tral Japan, inferred from dense seismic observations, Geophys. J. Int., 185, 265-282, doi:10.1111/j.1365-246X.2011.04938.x, 2011.

Maeda, Y., M. Takeo, and T. Ohminato, A waveform inversion including tilt: method and simple tests, Geophys. J. Int., 184, 907-918, doi:10.1111/j.1365-246X.2010.04892.x, 2011.

Nakamura, T., H. Takenaka, T. Okamoto, and Y. Kaneda, A study of the nite difference solution for 3D seismic wave elds near a uid-solid interface, J. Seismol. Soc. Jpn., 63, 189-196, 2011.

Nakano, M. and H. Kumagai, Waveform inversion of volcano-seismic signals assuming possible source geometries, Geophys. Res. Lett., 32, L12302, doi:10.1029/2005GL022666, 2005.

Nakano, M., H. Kumagai, and B. A. Chouet, Source mechanism of long-period events at Kusatsu-Shirane Volcano, Japan, inferred from waveform inversion of the effective excitation functions, J. Volcanol. Geotherm. Res., 122, 149-164, 2003.

Ohminato, T., Characteristics and source modeling of broadband seismic signals associated with the hydrothermal system at SatsumaIwojima volcano, Japan, J. Volcanol. Geotherm. Res., 158, 467-490, doi:10.1016/j.jvolgeores.2006.08.004, 2006.

Ohminato, T. and B. A. Chouet, A free-surface boundary condition for including 3D topography in the nite-difference method, Bull. Seismol. Soc. Am., 87, 494-515, 1997.

Ohminato, T., B. A. Chouet, P. Dawson, and S. Kedar, Waveform inversion of very long period impulsive signals associated with magmatic injec- tion beneath Kilauea Volcano, Hawaii, J. Geophys. Res., 103, 2383923862, 1998.

Okamoto, T. and H. Takenaka, Fluid-solid boundary implementation in the velocity-stress nite-difference method, J. Seismol. Soc. Jpn., 57, 355364, 2005.

Petukhin, A., T. Iwata, and T. Kagawa, Study on the effect of the oceanic water layer on strong ground motion simulations, Earth Planets Space, 62, 621-630, 2010.

Takeo, M., Near- eld synthetic seismograms taking into account the effects of anelasticity-The effects of anelastic attenuation on seismograms caused by a sedimentary layer, Pap. Meteorol. Geophys., 36, 245257, 1985.

Yamamoto, M., H. Kawakatsu, K. Yomogida, and J. Koyama, Long-period (12 sec) volcanic tremor observed at Usu 2000 eruption: Seismological detection of a deep magma plumbing system, Geophys. Res. Lett., 29, 1329-1332, doi:10.1029/2001GL013996, 2002.

Zlotnicki, J., Y. Sasai, J. P. Toutain, E. U. Villacorte, A. Bernard, J. P. Sabit, J. M. Gordon Jr, E. G. Corpuz, M. Harada, J. T. Punongbayan, H. Hase, and T. Nagao, Combined electromagnetic, geochemical and thermal surveys of Taal volcano (Philippines) during the period 2005-2006, Bull. Volcanol., 71, 29-47, doi:10.1007/s00445-008-0205-2, 2009.

Y. Maeda (e-mail: ymaeda@bosai.go.jp) and H. Kumagai 